\title{
Emotional intelligence in schizophrenia
}

\author{
Kimmy S. Kee a,b,c,*, William P. Horan ${ }^{\text {b,c }}$, Peter Salovey ${ }^{\text {d }}$, Robert S. Kern ${ }^{\text {b,c }}$, Mark J. Sergi ${ }^{c, e}$, \\ Alan P. Fiske ${ }^{\mathrm{f}}$, Junghee Lee ${ }^{\mathrm{b}, \mathrm{c}}$, Kenneth L. Subotnik ${ }^{\mathrm{b}}$, Keith Nuechterlein ${ }^{\mathrm{b}, \mathrm{g}}$, \\ Catherine A. Sugar ${ }^{\mathrm{h}}$, Michael F. Green ${ }^{\mathrm{b}, \mathrm{c}}$ \\ a Psychology Program, California State University Channel Islands, USA \\ ${ }^{\mathrm{b}}$ Department of Psychiatry and Biobehavioral Sciences, Geffen School of Medicine at University of California, Los Angeles, USA \\ c VA Greater Los Angeles Healthcare System VISN22 MIRECC, USA \\ ' Yale College Dean's Office and Department of Psychology, Yale University, USA \\ e Department of Psychology, California State University, Northridge, USA \\ ${ }^{\mathrm{f}}$ Department of Anthropology; Center for Culture, Brain, and Development; and Center for Behavior, Evolution, and Culture, University of California, Los Angeles, USA \\ g Department of Psychology, University of California, Los Angeles, USA \\ h Department of Biostatistics, School of Public Health, University of California, Los Angeles, USA
}

\section{A R T I C L E I N F O}

\section{Article history:}

Received 20 February 2008

Received in revised form 30 July 2008

Accepted 1 August 2008

Available online 21 September 2008

\section{Keywords:}

Schizophrenia

Emotional intelligence

Emotion processing

Social cognition

\begin{abstract}
A B S T R A C T
Background: Deficits in emotion perception have been extensively documented in schizophrenia and are associated with poor psychosocial functioning. However, little is known about other aspects of emotion processing that are critical for adaptive functioning. The current study assessed schizophrenia patients' performance on a theoretically-based, wellvalidated, multidimensional measure of emotional intelligence, the Mayer-Salovey-Caruso Emotional Intelligence Test (Mayer, J.D., Salovey, P., Caruso, D.R., 2002. Mayer-Salovey-Caruso Emotional Intelligence Test (MSCEIT): User's Manual. Multi-Health Systems, Inc., Toronto, Ontario).

Methods: 50 schizophrenia outpatients and 39 non-psychiatric controls completed the MSCEIT, a performance measure comprised of subtests that assess four components (branches) of emotional intelligence: Identifying, Using, Understanding, and Managing Emotions. Among patients, associations between MSCEIT scores and measures of clinical symptoms as well as functional outcome were evaluated.

Results: The MSCEIT demonstrated good psychometric properties in both groups. Schizophrenia patients performed significantly worse than controls on the total MSCEIT score, and on three of the four subtests: Identifying, Understanding, and Managing Emotions. Among patients, lower MSCEIT scores significantly correlated with higher negative and disorganized symptoms, as well as worse community functioning.

Conclusions: The MSCEIT is a useful tool for investigating emotion processing in schizophrenia. Individuals with schizophrenia demonstrate deficits across multiple domains of emotion processing. These deficits have significant links with clinical symptoms of schizophrenia and with how patients function in their daily lives. Further research is required to understand the links between emotional intelligence, clinical symptoms, and functional outcome in schizophrenia.
\end{abstract}

(c) 2008 Elsevier B.V. All rights reserved.

\footnotetext{
* Corresponding author. VA Greater Los Angeles Healthcare System, 11301 Wilshire Blvd. (MIRECC 210A), Building 210, Los Angeles, CA 90073, USA. Tel.: +1310 478 4711x49221; fax: +1 3102684056 .

E-mail address: kee@ucla.edu (K.S. Kee).
}

\section{Introduction}

Disturbances in social cognition are increasingly recognized as common, clinically important features of schizophrenia (Penn et al., 2006). Social cognition encompasses a 
wide range of processes that are required for adaptive functioning, including perceiving, interpreting, and responding to the behaviors, emotions, and intentions of others (Brothers, 1990; Fiske and Taylor, 1991; Adolphs, 2001). In basic behavioral science research, the domain of social cognition encompasses a broad array of social learning, perceptual, and cognitive phenomena, such as vicarious learning, perceived self-efficacy, locus of control, selfregulation (Mischel, 1973), and human agency (Bandura, 2006). Research on social cognition in schizophrenia has centered on five systems: emotion processing, social perception, social knowledge, theory of mind, and attributional style (Green et al., 2005). The clinical importance of disturbances in these areas is demonstrated by their significant associations with various aspects of poor functional outcome, such as success in psychosocial rehabilitation programs and functioning in the areas of social relationships, independent living, and work/school endeavors (Couture et al., 2006). Furthermore, there is emerging evidence that social cognitive deficits mediate the wellestablished link between basic (non-social) cognition and psychosocial functioning in schizophrenia (Addington et al., 2006; Brekke et al., 2005; Sergi et al., 2006; Vauth et al., 2004). Thus, a deeper understanding of the scope and clinical correlates of social cognitive deficits in schizophrenia can help identify rational targets for new treatments to improve emotion processing and functional outcome.

The most extensively studied aspect of social cognition in schizophrenia is emotion processing, which refers broadly to perceiving, understanding, and managing emotion in oneself and others (e.g., Green et al., in press). Schizophrenia research has focused almost exclusively on emotion perception (the ability to detect emotion information from others' facial expressions), vocal inflections, or a combination of these cues. This is most commonly assessed by asking research participants to identify prototypical emotions expressed in static pictures of faces or recordings of voices (e.g., happy, sad, angry, afraid, surprised, disgusted, ashamed). Schizophrenia patients have repeatedly shown poorer performance than healthy controls on these types of tasks (for a review see Edwards et al., 2002). Among patients, performance on these types of tasks has sometimes, but not consistently, shown significant relationships to certain clinical symptoms (Edwards et al., 2002; Bozikas et al., 2004). However, impairment on these tasks is consistently associated with poor community functioning, particularly in the areas of independent living and work functioning (Kee et al., 2003; Couture et al., 2006).

Although there is general agreement that disturbances in emotion processing are an important element of the social cognitive deficits of schizophrenia, research in this area is limited by two related issues, one conceptual and one methodological. First, although the concept of emotion processing is quite broad, schizophrenia research has narrowly focused on emotion perception. This is primarily attributable to the fact that until recently basic social cognitive and affective scientists have not developed or tested theoretical models of the key components of emotion processing. Second, schizophrenia researchers have often used idiosyncratic emotion processing measures that have substantial psychometric limitations (e.g., ceiling effects, low reliability) and questionable ecological validity. A theoretically-based, psychometrically sound measure that assesses the broader construct of emotion processing would greatly facilitate research in this area.

In recent years, social cognitive and affective scientists have made considerable advances in defining key emotion processing components and developing instruments to assess them. One particularly influential framework is emotional intelligence (EI) theory developed by Mayer et al. (Mayer et al., 2001; Salovey and Grewal, 2005; Salovey and Mayer, 1990). The EI model comprises four interrelated emotion processing abilities or skill "branches": (a) Perceiving emotions: the ability to perceive accurately, appraise, and express emotion; (b) Using Emotions: the ability to access and/or generate feelings when they facilitate thought; (c) Understanding Emotions: the ability to understand emotion language and knowledge about emotions; (d) Managing Emotions: the ability to regulate emotions to promote emotional and intellectual growth in oneself or other people (Mayer and Salovey, 1997). Branches 1 and 3 involve reasoning about emotions, whereas branches 2 and 4 involve Using Emotions adaptively.

To evaluate this model, Mayer et al. developed a multidimensional, task-based, ability measure called the MayerSalovey-Caruso Emotional Intelligence Test 2.0 (MSCEIT; Mayer et al., 2002). The MSCEIT and its predecessors are based on the idea that emotional intelligence involves problem solving with and about emotions. The test yields total and branch scores, based on either consensus or expert scoring, and has a factor structure consistent with the fourbranch model (Brackett and Mayer, 2001). The reliability and validity of the MSCEIT have been extensively documented in non-clinical populations (Mayer et al., 2003). For example, MSCEIT data collected from 2112 adults indicated that this test has very good internal consistency, with an alpha coefficient of .91 for the total scale, and short-term (two week) test-retest reliability $(r=0.86$; Brackett and Mayer, 2001). It assesses abilities that are separable from traditional measures of general intellectual ability, personality traits, and self-report measures of emotional competence (e.g., Brackett and Mayer, 2003; Lopes et al., 2003). In addition, higher MSCEIT scores are associated with higher levels of adaptive functioning across a variety of domains, including relationships with parents, friends, and romantic partners (Lopes et al., 2004, 2005), interpersonal effectiveness (Rode et al., 2007), success in the workplace (Lopes et al., 2006), academic success (Brackett et al., 2004), and various health-related outcomes (see Mayer et al., 2008 for a thorough summary). Importantly, the MSCEIT demonstrates good incremental validity, showing relationships with various indices of adaptive functioning above and beyond the effects of general IQ or personality measures (e.g., Brackett and Mayer, 2003; Mayer et al., 2003).

Although promising in its potential, we know almost nothing about the utility of the MSCEIT for assessing emotion processing in individuals with severe mental illnesses. One study by Eack et al. (2007) utilized the MSCEIT to examine the efficacy of Cognitive Enhancement Therapy on social cognition in schizophrenia. However, that study did not include healthy comparisons subjects or examine whether MSCEIT scores were related to clinically relevant features of schizophrenia. 
The current study was designed to address three research questions:

1. Do community-dwelling schizophrenia outpatients differ from non-psychiatric controls in their level of performance across the broad scope of emotion processes as assessed using the MSCEIT?

2. Are the MSCEIT scores associated with clinical symptoms?

3. Are the MSCEIT scores associated with community functioning in patients?

Based on the existing literature on emotion perception, we expected patients to perform worse than controls on the Identifying Emotions branch of the MSCEIT. The emotional processes that are measured by the other MSCEIT branches have not previously been examined in schizophrenia. Thus, we viewed these examinations as exploratory.

\section{Methods}

\subsection{Participants}

Fifty chronic schizophrenia outpatients and 39 nonpatient controls were part of a project within the UCLA Center for Neurocognition and Emotion in Schizophrenia (K. H. Nuechterlein, Center P.I. and M. F. Green, Project P.I.). All patients met criteria for schizophrenia disorder based on the Structured Clinical Interview for DSM-IV (SCID; First et al., 1996a).

Non-patient controls were screened with the SCID-I, relevant sections of the SCID-II (First et al., 1996b), and the positive symptom section of the Structured Interview for Prodromal States (SIPS; Miller et al., 1999), and were excluded if they met criteria for a history of any DSM-IV Axis I Psychotic Disorder, any schizophrenia-spectrum disorder Axis II Disorder (i.e., paranoid, schizotypal, schizoid, and/or avoidant personality disorder), or any of the positive symptoms (P1P5) of the SIPS rated "3" or higher during the past year. Potential control subjects were also excluded for a history of any of the following: major depression disorder that was ongoing, recurrent, or a single episode that lasted longer than one year; bipolar disorder; obsessive-compulsive disorder; post-traumatic stress disorder; and alcohol/substance dependency (lifetime).

Additional exclusion criteria for all participants included: active substance use disorder in the past six months, identifiable neurological disorder, significant head injury, or pregnancy at the time of recruitment. Diagnostic interviewers were trained to a minimum Kappa of 0.75 for rating psychotic and mood symptoms. Informed consent was obtained from each participant using both written materials and verbal description. This study was approved by the Institutional Review Board of University of California Los Angeles.

\subsection{Measures and procedures}

All participants received the assessment of emotion processing with the MSCEIT. In addition, data were collected on general psychosocial outcome and psychiatric symptoms in the schizophrenia group. Testing for all measures took up to $120 \mathrm{~min}$ for schizophrenia patients and $30 \mathrm{~min}$ for normal controls (who did not receive functional outcome assess- ments or ratings of psychiatric symptoms). The test battery included the following:

\subsubsection{Emotional intelligence test}

The MSCEIT is a self-report instrument that consists of 141 items and 8 ability subscales, which assess four components (branches) of emotion processing. The first branch, Identifying Emotions, has 2 subscales measuring emotion perception in faces and pictures. For example, to identify emotions from a face, subjects were asked to indicate the degree to which each of five feelings (e.g., happiness, sadness, fear, anger, and disgust) was expressed by a color photograph of a human face.

The second branch, Using Emotions (to facilitate cognition), contains 2 subscales examining how mood enhances thinking and reasoning, and which emotions are associated with which sensations. For example, subjects were asked to evaluate the usefulness of 3 different emotions (e.g., happiness, surprise, and sadness) that best assisted a specific cognitive task and behavior, such as: "What mood(s) might be helpful to feel when figuring out what caused a fight among three young children?"

The third branch, Understanding Emotions, has 2 subscales that measure the ability to comprehend emotional information, including blends and changes between and among emotions. For example, to assess understanding of the progressions of emotion, participants were asked to select which one of five emotions best described a situation, such as: "Matt had been hurt by one of his friends and was feeling angry. Matt told his friend how he felt, and when the friend did it again, Matt became ___.." The choices were 1 = angry; 2 = fearful; 3 = very annoyed; 4 = worried; and 5 = enraged.

The last branch, Managing Emotions, has 2 subscales that examine the regulation of emotions in oneself and in one's relationships with others. These subscales include vignettes of various situations, along with ways to cope with the emotions depicted in these vignettes. Subjects were required to indicate the effectiveness of each solution, ranging from 1 (very ineffective) to 5 (very effective).

The MSCEIT was administered to schizophrenia patients and normal controls individually. Each participant received a booklet that contained all necessary instructions, test items, and responses. Responses included 5-point Likert ratings with specific anchor points for some items and a 5-item multiplechoice format for others. These responses could be scored either by using the general consensus of lay persons or expert norms, with a mean scale of $100(S D=15)$. For the current study, the MSCEIT scores were derived using the general consensus approach (see Mayer et al., 2003 for more information about these scoring alternatives and their psychometric properties).

\subsubsection{Clinical rating scales}

Psychiatric symptoms were assessed using the Scale for the Assessment of Negative Symptoms (SANS; Andreasen, 1984a) and Scale for the Assessment of Positive Symptoms (SAPS; Andreasen, 1984b). All raters received extensive training on these measures to ensure a minimum Intraclass Coefficient (ICC) of 0.75 . From the SANS, four global subscale scores (rated from 0 (symptom absent) to 5 (symptom severe) were used in the current study: affective flattening, alogia, 
Table 1

Demographic data

\begin{tabular}{|c|c|c|c|}
\hline & $\begin{array}{l}\text { Schizophrenia } \\
\text { patients }\end{array}$ & Controls & Statistics \\
\hline $\bar{N}$ & 50 & 39 & \\
\hline Age $(M, \mathrm{SD})$ & 34.37 (7.69) & $32.97(5.17)$ & $t(86)=1.01$ \\
\hline Sex (\% male) & 62 & 65 & $\chi^{2}(1, N=89)=0.27$ \\
\hline Education $(M, \mathrm{SD})$ & $14.02(1.60)$ & $14.28(1.75)$ & $t(86)=-.73$ \\
\hline $\begin{array}{l}\text { Education - } \\
\text { parents }(M, \mathrm{SD})\end{array}$ & $15.18(2.98)$ & $14.36(2.95)$ & $t(86)=1.29$ \\
\hline Ethnicity $(N)$ & & & $\chi^{2}(4, N=89)=2.85$ \\
\hline Caucasian & 31 & 28 & \\
\hline African American & 9 & 8 & \\
\hline Hispanic & 0 & 0 & \\
\hline Asian & 5 & 2 & \\
\hline Other & 5 & 1 & \\
\hline Marital status $(N)$ & & & $\chi^{2}(3, N=89)=0.07$ \\
\hline Never married & 37 & 30 & \\
\hline Currently married & 7 & 5 & \\
\hline Divorced/separated & 4 & 3 & \\
\hline Widowed & 1 & 1 & \\
\hline
\end{tabular}

${ }^{*} p<.05 ; 2$-tailed $t$-tests.

anhedonia-asociality, and avolition-apathy. The SANS Attention scale was not included in the current analyses given findings suggesting that this scale is not conceptually related to the negative symptom complex (e.g., Blanchard and Cohen, 2006). From the SAPS, global scores from the hallucinations, delusions, bizarre behavior, and thought disorder subscales were used.

\subsubsection{Psychosocial outcome measures}

General psychosocial functioning was assessed using two measures: the Role Functioning Scale (RFS; McPheeters, 1984) and the UCLA Social Attainment Scale (SAS; Goldstein, 1978). The Role Functioning Scale (RFS) measures four major domains of participants' functioning in everyday life. The domains are work productivity, independent living/self-care, relationships with family and spouse, and relationships with friends, each rated on specific anchor points ranging from 1 (severely limited functioning) to 7 (optimal functioning). The RFS has sound psychometric properties (Goodman et al., 1993) and has been used widely in service outcome studies in schizophrenia (e.g., Brekke and Long, 2000; Brekke et al., 1997; Green and Gracely, 1987). Examples of anchor points for the family relationships component include: $1=$ no contact with any family members or severely deviant behaviors within family network; 4 = relationships were often minimal and fluctuate in quality (contact once a month); and 7 = positive relationships with several family members and assertively contributed to these relationships. For the current study, the RFS ratings were completed based on the Community Assessment of Functioning, which is a comprehensive semi-structure interview that assesses several aspects of community functioning.

The 7-item UCLA Social Attainment Scale (SAS) assesses patients' psychosocial adjustment in the following domains: same sex relationship, romantic relationship, leadership, dating history, sexual experience, participation in outside activities, and participation in organizations. Each item was rated from 1 (poor) to 5 (superior). For this study, a composite of current psychosocial adjustment index was calculated based on the seven domains.

\section{Results}

\subsection{Participant characteristics}

Demographic data for schizophrenia patients and control participants are summarized in Table 1 . The groups did not significantly differ in age, sex, education level, parental education, ethnicity, and marital status. The schizophrenia outpatients in this study exhibited mild levels of symptoms on the SANS total score $(M=0.95, \mathrm{SD}=0.79)$ and SAPS total score $(M=1.79, S D=1.05)$. The patients' average length of illness was 10.20 years $(S D=5.30)$.

\subsection{Test of reliability}

The internal consistency for each of the MSCEIT branches and the total score was computed separately for the two groups. Cronbach's alphas revealed generally high reliability coefficients for most MSCEIT branches (Identifying Emotions, $\alpha=0.86$; Using Emotions, $\alpha=0.83$; Understanding Emotions, $\alpha=0.82$; and Managing Emotions, $\alpha=0.72$ ) and the total score $(\alpha=0.93)$ in the schizophrenia sample. Although reliability estimates for control subjects were somewhat lower, they were within adequate range (Identifying Emotions, $\alpha=0.89$; Using Emotions, $\alpha=0.78$; Understanding Emotions, $\alpha=0.68$; and Managing Emotions, $\alpha=0.67$ ), with the total score showing the highest level $(\alpha=0.90)$. Also, the test for the distribution of the MSCEIT total score (skewness = -0.10 , standard error $=0.34$ ) in the patient group was fairly comparable to that of the control group score (skewness= -0.40 , standard error $=0.39$ ), indicating relatively normal distributions for both groups.

\subsection{Performance on the emotional intelligence test}

MSCEIT scores are presented in Fig. 1 and Table 2. The main analyses evaluated group differences of performance across the MSCEIT branches, using the SAS PROC MIXED procedure with a two-way mixed model regression. Participant group (schizophrenia patients, controls) was the independent grouping variable, the MSCEIT branch was a within-subjects repeated measures factor with 4 levels (Identifying Emotions, Using

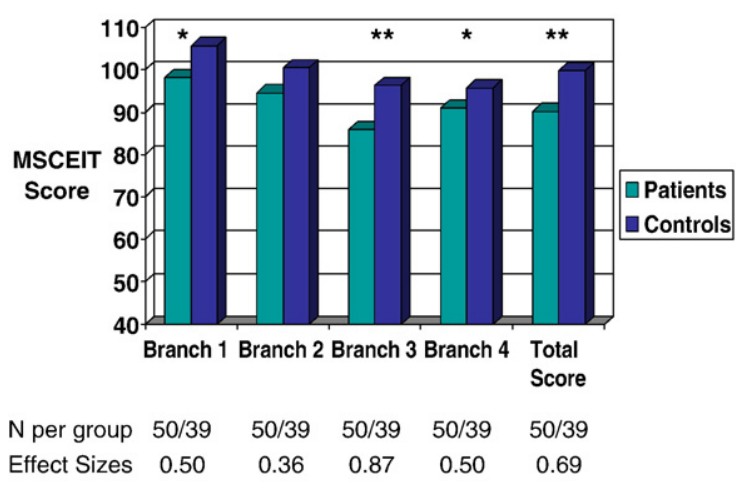

${ }^{\star} \mathrm{p}<.05 ;{ }^{\star \star} \mathrm{p}<.01 ; 2$-tailed $\mathrm{t}-$ Tests

Fig. 1. Group differences on MSCEIT measures: chronic patients versus controls. 
Table 2

Group differences on MSCEIT measures

\begin{tabular}{|c|c|c|c|c|c|}
\hline \multirow[t]{2}{*}{ MSCEIT branches } & \multirow[t]{2}{*}{ Subscales } & \multirow{2}{*}{$\frac{\text { Schizophrenia patients }}{(n=50)}$} & \multirow{2}{*}{$\frac{\text { Controls }}{(n=39)}$} & \multirow[t]{2}{*}{ t-tests } & \multirow[t]{2}{*}{$d$} \\
\hline & & & & & \\
\hline \multirow[t]{2}{*}{ Branch 1: Identifying Emotions } & Faces & $106.91(22.98)$ & $116.95(24.46)$ & $-1.99^{*}$ & 0.42 \\
\hline & Pictures & $94.03(9.30)$ & $103.36(14.00)$ & $-3.59^{* *}$ & 0.81 \\
\hline \multirow[t]{2}{*}{ Branch 2: Facilitating Emotions } & Facilitation & 97.55 (19.68) & $101.33(15.03)$ & -0.99 & 0.21 \\
\hline & Sensations & $93.41(12.12)$ & $95.08(10.48)$ & -0.69 & 0.15 \\
\hline \multirow[t]{2}{*}{ Branch 3: Understanding Emotions } & Changes & $86.96(12.66)$ & $97.08(12.04)$ & $-3.84^{* *}$ & 0.82 \\
\hline & Blends & $90.04(12.49)$ & $97.50(10.07)$ & $-3.04^{* *}$ & 0.65 \\
\hline \multirow[t]{2}{*}{ Branch 4: Managing Emotions } & Emotion Management & 92.68 (10.29) & $96.94(9.89)$ & $-1.96^{*}$ & 0.42 \\
\hline & Emotional Relationships & $90.24(9.45)$ & $95.51(8.96)$ & $-2.67^{*}$ & 0.57 \\
\hline
\end{tabular}

${ }^{*} p<.05 ;{ }^{* *} p<.01 ; 2$-tailed $t$-tests.

Emotions, Understanding Emotions, and Managing Emotions), and subject was included as a random effect to account for nonindependence (the use of multiple records from individuals).

The regression analyses revealed a significant group effect $(F(1,87)=10.50, p<.002)$ and a significant branch effect $(F(3,87)=$ $15.16, p<.0001)$, but there was no significant group $\mathrm{x}$ branch interaction. For the MSCEIT total score, the schizophrenia sample performed significantly worse than controls, $t(87)=-3.21$, $p<.002$. Also, schizophrenia patients demonstrated significantly poorer performance compared to controls on 3 of the 4 MSCEIT branches: Identifying Emotions, Understanding Emotions, and Managing Emotions (all ps<.02). For Using Emotions, the schizophrenia group displayed a tendency to perform worse than controls at a trend level, $t(87)=-1.70, p<.09$.

Analyses of group differences on the subscales that comprise the main MSCEIT branches indicated that the patients performed worse than controls on 6 of the 8 subscales (i.e., faces, pictures, changes, blends, emotion management, and emotional relationships). These 6 subscales were components of the 3 MSCEIT branches that yielded the significant group differences described above. Patients did not significantly differ on the facilitation or sensations subscale from the Using Emotions branch. The significant patient-control group differences yielded medium to large effect sizes, with $d s$ ranging from 0.36 to 0.87 (Cohen, 1988).

\subsection{Clinical correlates}

We next examined whether the MSCEIT total and branch scores were related to psychiatric symptoms in the schizophrenia sample. As shown in Table 3, significant negative associations were found for all of the components of the

Table 3

Correlations among MSCEIT measures, SANS, and SAPS

\begin{tabular}{llllll}
\hline & Branch 1 & Branch 2 & Branch 3 & Branch 4 & Total score \\
\hline Affective flattening & $-0.36^{*}$ & $-0.30^{*}$ & $-0.30^{*}$ & $-0.43^{* *}$ & $-0.41^{* *}$ \\
Alogia & -0.14 & -0.18 & -0.10 & -0.27 & -0.18 \\
Avolition & $-0.38^{* *}$ & -0.21 & -0.15 & -0.24 & $-0.29^{*}$ \\
Anhedonia & $-0.42^{* *}$ & -0.23 & -0.20 & -0.27 & $-0.33^{*}$ \\
Total SANS Score & $-0.40^{* *}$ & -0.28 & -0.23 & $-0.37^{* *}$ & $-0.37^{* *}$ \\
Hallucinations & -0.06 & -0.20 & -0.15 & -0.21 & -0.17 \\
Delusions & 0.02 & -0.11 & -0.02 & -0.03 & -0.03 \\
Bizarre behavior & $-0.35^{*}$ & -0.26 & 0.02 & -0.10 & -0.24 \\
Thought disorders & -0.16 & -0.22 & $-0.47^{* *}$ & -0.19 & $-0.32^{*}$ \\
Total SAPS Score & -0.16 & $-0.30^{*}$ & $-0.31^{*}$ & -0.23 & $-0.29^{*}$ \\
\hline
\end{tabular}

${ }^{*} p<.05 ;{ }^{* *} p<.01 ; 2$-tailed.
MSCEIT and affective flattening of the SANS. Identifying Emotions and MSCEIT total scores were also significantly negatively correlated with the SANS avolition-apathy and anhedonia-asociality subscales. In addition, Identifying Emotions, Managing Emotions, and MSCEIT total scores were significantly negatively related to the SANS total score. The magnitudes of all of the significant correlations were small to medium.

Table 3 also shows significant negative associations between components of the MSCEIT and a few positive symptoms: Identifying Emotions with bizarre behavior, Using Emotions with the SAPS total score, and Understanding Emotions and the MSCEIT total score with thought disorders and the SAPS total score. The magnitudes of these correlations were also small to medium.

\subsection{Psychosocial outcome correlates}

Table 4 presents correlations between performance on the MSCEIT and domains of general psychosocial functioning in the schizophrenia sample. There were several statistically significant associations: Identifying Emotions with work productivity; all of the components of the MSCEIT with independent living/self-care; Identifying Emotions with relationships with family and spouse; and Identifying Emotions and Understanding Emotions with psychosocial adjustment. The magnitudes for the above correlations were small to medium, and in these correlations lower levels of emotional intelligence processes were associated with poorer psychosocial outcome in schizophrenia.

\subsection{Supplemental analyses}

Because components of the MSCEIT showed significant associations with independent living/self-care and also with

Table 4

Correlations among MSCEIT measures and general psychosocial functioning

\begin{tabular}{|c|c|c|c|c|c|}
\hline & Branch 1 & Branch 2 & Branch 3 & Branch 4 & Total score \\
\hline Work productivity & $0.34^{*}$ & 0.23 & 0.08 & 0.24 & 0.26 \\
\hline $\begin{array}{l}\text { Independent } \\
\text { living/self-care }\end{array}$ & $0.43^{* *}$ & $0.33^{*}$ & $0.31^{*}$ & $0.36^{*}$ & $0.41^{* *}$ \\
\hline $\begin{array}{l}\text { Family network } \\
\text { relationships }\end{array}$ & $0.35^{*}$ & 0.06 & 0.27 & 0.18 & 0.27 \\
\hline Social relationships & 0.25 & 0.22 & 0.12 & 0.21 & 0.23 \\
\hline Social adjustment & $0.33^{*}$ & 0.11 & $0.30^{*}$ & 0.10 & 0.25 \\
\hline
\end{tabular}

${ }^{*} p<.05 ;{ }^{* *} p<.01 ; 2$-tailed. 
SANS and SAPS subscales, we performed a mediator analysis using a sequence of regression models as suggested by Baron and Kenny (1986) to determine whether the association between emotional intelligence and independent living/selfcare outcome was explained by psychiatric symptoms. A mediation effect would be present if: 1) there is a strong correlation between the predictor variable (i.e., the MSCEIT total score) and the outcome variable (i.e., independent living/ self-care), 2) there is a significant relationship between the predictor variable and the mediator (i.e., SAPS or SANS total score), 3) there is a significant relationship between the mediator and the outcome after adjustment for the predictor variable, and 4) the relationship between the predictor and outcome is no longer significant (or is significantly reduced) when the mediator is entered into the model.

The results of the mediator analysis revealed that negative symptoms mediated the effect of emotional intelligence on independent living/self-care. The MSCEIT total score alone was significantly related to both the SANS total score $(\beta=-0.027$, $\mathrm{SE}=0.010, p=.0096)$ and to the independent living/self-care score $(\beta=0.039, \mathrm{SE}=0.013, p=.0036)$. When the total scores from the MSCEIT and SANS were used jointly to predict independent living/self-care, the SANS total score was significant $(\beta=0.668, \mathrm{SE}=0.165, p=.0002)$, but the MSCEIT total score was reduced to a trend level, with the magnitude of its coefficient cut nearly in half $(\beta=0.021, \mathrm{SE}=0.012, p=.0797)$. As a follow-up analysis, we conducted Sobel's test (Aroian version, cf. Baron and Kenny, 1986) to examine the significance of the mediated path (that is, for the indirect effect of emotional intelligence on independent living/self-care via negative symptoms). The resulting $Z$-score of 2.20 was significant $(p=.0280)$, and the positive sign indicates that as emotional intelligence (mediated by negative symptoms) increases, so does independent living/self-care skills, as expected. In addition, the MSCEIT total score accounted for only $4.4 \%$ of the variance in independent living/self-care skills above and beyond the $34.8 \%$ of the variance accounted for by negative symptoms alone, whereas the SANS total score accounted for an additional $22.2 \%$ of variance above and beyond that accounted for by the MSCEIT total score alone, reinforcing our conclusions. We did not find a mediation effect for positive symptoms.

\section{Discussion}

In the present study, we tested a broad range of emotion processing abilities in individuals with schizophrenia and nonpsychiatric controls using a theory-based multidimensional assessment of emotional intelligence, the Mayer-SaloveyCaruso Emotional Intelligence Test (MSCEIT; Mayer et al., 2002). The MSCEIT demonstrated strong internal consistency in the schizophrenia sample and adequate consistency in a sample of control subjects, suggesting that this measure is suitable for use in schizophrenia research. Although previous studies of emotion processing in schizophrenia have focused almost exclusively on the perception of emotion, the current results suggest that patients display a considerably broader range of impairment across multiple emotion processing abilities. Furthermore, these abilities are meaningfully associated with varied aspects of patients' functioning in the community.

For the first research question concerning between-group comparisons on the MSCEIT, we replicated the previously reported deficits in identifying facial emotional expression (a subscale of Branch 1) in schizophrenia patients compared to normal controls (e.g., Addington and Addington, 1998; Edwards et al., 2002; Horan and Blanchard, 2003; Kee et al., 2004; Mandal et al., 1998; Streit et al., 1997). In addition, we found clear patient versus control differences on two other domains of emotion processing, Understanding Emotions and Managing Emotions, as well as the overall MSCEIT score. These differences indicate that individuals with schizophrenia show impairments in their understanding of blends and changes between and among emotions, as well as their regulation of emotions in everyday life.

The current results demonstrating group differences on regulation of emotions are consistent with prior findings of Henry et al. (2007), who reported aberrant emotional expression (i.e., reduced amplification of an experienced emotion) in schizophrenia patients in comparison with control participants. Also, it has been suggested that individuals with schizophrenia fail to use positive emotional experience to enhance memory functioning compared to healthy participants (Herbener et al., 2007). Although our patient sample exhibited a tendency to perform worse than controls on Using Emotions (how well participants used different emotions to enhance cognition and behavior), this group difference was not statistically significant. Overall, the current study suggests that the inclusion of a broader assessment of emotion processes that extends beyond perception of emotion may be useful in understanding the full range of social cognitive deficits of schizophrenia.

Regarding the second research question, we evaluated associations between clinical symptoms and the broad scope of emotion processing abilities as assessed by the MSCEIT in the schizophrenia group. For negative symptoms, all of the MSCEIT components were significantly related to affective flattening. Interestingly, schizophrenia patients with clinically significant flat affect have also been found to demonstrate deficits in identifying happy and sad emotions, and show even greater impairment in differentiating the intensities within both these emotions compared to healthy subjects (Gur et al., 2006). However, an earlier study by Sweet et al. (1998) revealed that schizophrenia patients with blunted affect were not more impaired in their perception of emotions than non-blunted patients. In addition, although not as consistent, there were a few significant relationships between emotional intelligence processes and other negative symptoms measures, including avolition-apathy and anhedonia-asociality. Some early studies reported similar relationships (e.g., Lewis and Garver, 1995; Mueser et al., 1996). For positive symptoms, patients who exhibited higher levels of bizarre behavior, thought disorders, and the SAPS total score performed worse in certain aspects of emotional intelligence, in line with previous findings of Kohler et al. (2000), Poole et al. (2000), and Schneider et al. (1995). Thus, the current findings support associations between the social cognitive processes assessed by the MSCEIT and several aspects of clinical symptoms. Additional research on the link between different clinical symptoms and various aspects of social cognition is needed (Green et al., in press).

For the third research question, the MSCEIT total score and scores of the specific branches demonstrated significant associations with several aspects of functioning in the community among individuals with schizophrenia. For 
instance, Identifying Emotions was significantly correlated with psychosocial adjustment as well as role functioning in the community. We also uncovered multiple significant relationships across other domains of the MSCEIT and psychosocial outcome, suggesting that schizophrenia patients with lower levels of emotional intelligence processes exhibited poorer psychosocial adjustment and competence in the community. Overall, these relationships were fairly modest, but appeared to be somewhat stronger for independent living/self-care skills than other aspects of outcome. One possible reason for the observed relationships is that perhaps independent living/self-care settings place a greater emphasis on performing prescribed tasks and achieving set goals. Patients' difficulty in processing emotion could lead to inappropriate responding, which might hinder their ability to execute the independent living/self-care task requirements. Hence, this psychosocial domain might be more sensitive to negative consequences arising from deficits in emotion processing compared with family or social relationships.

The significant associations between emotion processing and psychosocial outcome replicate and extend prior work in this area. Kee et al. (2003) demonstrated that the ability to perceive emotion might predict work functioning or independent living outcome one year later in a clinically stabilized schizophrenia outpatient sample. Mueser et al. (1996) reported that accurate identification of emotional stimuli was related to personal appearance and hygiene, whereas Penn et al. (1996) and Poole et al. (2000) reported relationships with neatness and appropriate clothing and appearance in schizophrenia. However, negative symptoms appeared to mediate the relationship between general emotional intelligence and living/self-care skills. This mediation could be partly attributable to content overlap between the SANS (particularly the avolition subscale) and the measures of independent live/social care skills (Horan et al., 2006). The effect was not found when positive symptoms were used as a mediator. Future studies may be useful in clarifying the relationships among emotional intelligence, psychiatric symptoms, and psychosocial outcome.

Some limitations of the current study should be noted. First, the measures of psychosocial functioning (i.e., the Role Functioning Scale; RFS and the UCLA Social Attainment Scale; SAS) are quite global, relying on objective indicators of frequency of engagement across a rather wide range of community activities. Including other "micro-level" psychosocial outcome (e.g., social skills or social competence) or more sensitive assessments (e.g., quality of relationships and activities engaged in) may reveal more specific patterns of relationship with emotional intelligence. Second, given the size of this sample and the exploratory nature of this study, we did not correct for multiple statistical tests, which could lead to Type 1 errors. Third, although we examined a stabilized community-dwelling sample, it is not clear whether the disturbances shown by the patients represented state or trait-level phenomenon, or were associated with factors such as illness chronicity or long-term exposure to antipsychotic medications. Studies of first episode and prodromal patients will help clarify the trait status of emotion processing deficits in schizophrenia. Fourth, a non-emotion processing task was not administered, as part of this protocol, leaving open the question of whether emotional intelligence plays a role in the relationships between other known predictors (e.g., neurocognition) and psychosocial outcome.

To our knowledge, the present study is the first to directly explore the use of a comprehensive, multidimensional instrument that allows evaluation of schizophrenia patients' performance on a broad range of emotion processes, comparing patients to well-matched controls. The current study encourages broader assessments of emotion processing than those typically used in social cognitive studies of schizophrenia. In future studies, it may useful for schizophrenia researchers to tap into rapidly expanding affective and social neuroscience literature to investigate the neural correlates of emotion processing disturbances (e.g., Adolphs, 2003; Lieberman, 2007; Ochner and Phelps, 2007; Reis et al., 2007). In addition, there is growing evidence that negative symptomatology and emotional intelligence are key components of poor functional outcome in schizophrenia, and specific intervention programs designed to ameliorate these factors may be potentially useful in helping people with schizophrenia achieve more satisfying levels of recovery and functioning in the community (e.g., Horan et al., 2008).

\section{Role of funding source}

Funding for this project was supported by the National Institute of Mental Health Center Grant P50 MH 66586 (K. Nuechterlein, P.I.). The NIMH had no further role in study design; in the collection, analysis and interpretation of data; in the writing of the report; and in the decision to submit the paper for publication.

\section{Contributors}

All authors contributed to and have approved the final manuscript.

\section{Conflict of interest}

All other authors declare that they have no conflicts of interest.

\section{Acknowledgements}

Funding for this project was supported by the National Institute of Mental Health Center Grant P50 MH 66586 (K. Nuechterlein, P.I.). The authors wish to thank Mike DeGroot, Robin Kite, Jeff Nishii, and Samantha Swain for their assistance in data collection and data management.

\section{References}

Addington, J., Addington, D., 1998. Facial affect recognition and information processing in schizophrenia and bipolar disorder. Schizophr. Res. 32, 171-181.

Addington, J., Saeedi, H., Addington, D., 2006. Facial affect recognition: a mediator between cognitive and social functioning in schizophrenia? Schizophr. Res, 85, 142-150.

Adolphs, R., 2001. The neurobiology of social cognition. Curr. Opin. Neurobiol. $11,231-239$

Adolphs, R., 2003. Cognitive neuroscience of human social behaviour. Nat. Rev. Neurosci. 4 (3), 165-178.

Andreasen, N.C., 1984a. Scale for the Assessment of Positive Symptoms (SAPS). University of Iowa, Iowa City, IA.

Andreasen, N.C., 1984b. Scale for the Assessment of Negative Symptoms (SANS). University of Iowa, Iowa City, IA

Bandura, A., 2006. Toward a psychology of human agency. Perspect. Psychol. Sci. 1 (2), 164-180.

Baron, R.M., Kenny, D.A., 1986. The moderator-mediator variable distinction in social psychological research: conceptual, strategic, and statistical considerations. J. Pers. Soc. Psychol. 51 (6), 1173-1182.

Blanchard, J.J., Cohen, A.S., 2006. The structure of negative symptoms within schizophrenia: implications for assessment. Schizophr. Bull. 32, 238-245.

Bozikas, V.P., Kosmidis, M.H., Anezoulaki, D., Giannakou, M., Karavatos, A., 2004. Relationship of affect recognition with psychopathology and cognitive performance in schizophrenia. J. Int. Neuropsychol. Soc. 10, 549-558.

Brackett, M., Mayer, J.D., 2001. Measures of emotional intelligence. Poster Presented at the Third Annual Positive Psychology Summit. Washington, DC. 
Brackett, M.A., Mayer, J.D., 2003. Convergent, discriminant, and incremental validity of competing measures of emotional intelligence. Pers. Soc. Psychol. Bull. 29, 1147-1158.

Brackett, M.A., Mayer, J.D., Warner, R.M., 2004. Emotional intelligence and its expression in everyday behavior. Pers. Individ. Diff. 36, 1387-1402.

Brekke, J.S., Long, J.D., 2000. Community-based psychosocial rehabilitation and prospective change in functional, clinical, and subjective experience variables in schizophrenia. Schizophr. Bull. 26, 667-680.

Brekke, J.S., Long, J.D., Nesbitt, N., Sobel, E., 1997. The impact of service characteristics on functional outcomes from community support programs for persons with schizophrenia: a growth curve analysis. I. Consult. Clin. Psychol. 65, 464-475.

Brekke, J.S., Kay, D., Kee, K.S., Green, M.F., 2005. Biosocial pathways to functional outcome in schizophrenia. Schizophr. Res. 80 (2-3), 213-225.

Brothers, L., 1990. The neural basis of primate social communication. Motiv. Emot. 14, 81-91.

Cohen, I., 1988. Statistical Power Analysis for The Behavioral Sciences, second ed. Erlbaum, Hillsdale, NJ.

Couture, S.M., Penn, D.L., Roberts, D.L., 2006. The functional significance of social cognition in schizophrenia: a review. Schizophr. Bull. 32 (Suppl 1), S44-63.

Eack, S.M., Hogarty, G.E., Greenwald, D.P., Hogarty, S.S., Keshavan, M.S., 2007. Cognitive enhancement therapy improves emotional intelligence in early course schizophrenia: preliminary effects. Schizophr. Res. 89, 308-311.

Edwards, J., Jackson, H.J., Pattison, P.E., 2002. Emotion recognition via facial expression and affective prosody in schizophrenia: a methodological review. Clin. Psychol. Rev. 22 (6), 789-832.

First, M.B., Gibbons, M., Spitzer, R.L., Williams, J.B.W., 1996a. Users Guide for the Structured Clinical Interview for DSM-IV Axis I Disorders - Research Version - (SCID-I, Version 2.0, February 1996 Final Version). Biometric Research Department, New York.

First, M.B., Gibbons, M., Spitzer, R.L., Williams, J.B.W., Benjamin, L., 1996b. Structured Clinical Interview for DSM-IV Avis II Personality Disorders. Biometrics Research Department, New York.

Fiske, S.T., Taylor, S.E., 1991. Social Cognition, second ed. McGraw-Hill Book Co., New York.

Goldstein, M.J., 1978. Further data concerning the relation between premorbid adjustment and paranoid symptomatology. Schizophr. Bull. 4, 236-241.

Goodman, S.H., Sewell, D.R., Cooley, E.L., Leavitt, N., 1993. Assessing levels of adaptive functioning: the Role Functioning Scale. Community Ment Health J. 29, 119-131.

Green, R.S., Gracely, E.J., 1987. Selecting a rating scale for evaluating services to the chronically mentally ill. Community Ment. Health J. 23, 91-102.

Green, M.F., Olivier, B., Crawley, J.N., Penn, D.L., Silverstein, S., 2005. Social cognition in schizophrenia: recommendations from the MATRICS New Approaches Conference. Schizophr. Bull. 31, 882-887.

Green, M.F., Penn, D.L., Bentall, R., Carpenter, W.T., Gaebel, W., Gur, R.C., Kring, A.M., Park, S., Silverstein, S.M., Heinssen, R., in press. Social Cognition in Schizophrenia: an NIMH Workshop on Definitions, Assessment, and Research Opportunities. Schizophr. Bull.

Gur, R.E., Kohler, C.G., Ragland, J.D., Siegel, S.J., Lesko, K., Bilker, W.B., Gur, R.C., 2006. Flat affect in schizophrenia: relation to emotion processing and neurocognitive measures. Schizophr. Bull. 32 (2), 279-287.

Henry, J.D., Green, M.J., de Lucia, A., Restuccia, C., McDonald, S., O'Donnell, M., 2007. Emotion dysregulation in schizophrenia: reduced amplification of emotional expression is associated with emotional blunting. Schizophr. Res., 95, 197-204.

Herbener, E.S., Rosen, C., Khine, T., Sweeney, J.A., 2007. Failure of positive but not negative emotional valence to enhance memory in schizophrenia. J. Abnorm. Psychology 116 (1), 43-55.

Horan, W.P., Blanchard, J.J., 2003. Emotional responses to psychosocial stress in schizophrenia: the role of individual differences in affective traits and coping. Schizophr. Res. 20, 171-283.

Horan, W.P., Kring, A.M., Blanchard, J.J., 2006. Anhedonia in schizophrenia: a review of assessment strategies. Schizophr. Bull. 32, 259-273.

Horan, W.P., Kern, R.S., Green, M,F., Penn, D.L., 2008. Social cognition training for individuals with schizophrenia: emerging evidence. Am. J. Psychiatr. Rehabil. 11, 205-252.

Kee, K.S., Green, M.F., Mintz, J., Brekke, J.S., 2003. Is emotional processing a predictor of functional outcome in schizophrenia? Schizophr. Bull. 29(3), 487-497.

Kee, K.S., Horan, W.P., Mintz, J., Green, M.F., 2004. Do the siblings of schizophrenia patients demonstrate affect perception deficits? Schizophr. Res. 67, 87-94.

Kohler, C.G., Bilker, W., Hagendoorn, M., Gur, R.E., Gur, R.C., 2000. Emotion recognition deficit in schizophrenia: association with symptomatology and cognition. Biol. Psychiatry 48 (2), 127-136.
Lewis, S.F., Garver, D.L., 1995. Treatment and diagnostic subtype in facial affect recognition in schizophrenia. Psychiatry Res. 29, 5-11.

Lieberman, M.D., 2007. Social cognitive neuroscience: a review of core processes. Annu. Rev. Psychol. 58, 259-289.

Lopes, P.N., Salovey, P., Straus, R., 2003. Emotional intelligence, personality, and the perceived quality of social relationships. Pers. Individ. Differ. 35, 641-658.

Lopes, P.N., Brackett, M.A., Nezlek, J.B., Schutz, A., Sellin, I., Salovey, P., 2004. Emotional intelligence and social interaction. Pers. Soc. Psychol. Bull. 30, 1018-1034.

Lopes, P.N., Salovey, P., Cote, S., Beers, M., 2005. Emotion regulation abilities and the quality of social interaction. Emotion 5, 113-118.

Lopes, P.N., Grewal, D., Kadis, J., Gall, M., Salovey, P., 2006. Evidence that emotional intelligence is related to job performance and affect and attitudes at work. Psicothema 18, 132-138.

Mandal, M.K., Pandey, R.P., Prasad, A.B., 1998. Facial expressions of emotions and schizophrenia: a review. Schizophr. Bull., 24, 399-412.

Mayer, J.D., Salovey, P., 1997. What is emotional intelligence. In: Salovey, P., Sluyter, D. (Eds.), Emotional Development and Emotional Intelligence: Implications for Educators. Basic Books, New York, pp. 3-31.

Mayer, J.D., Salovey, P., Caruso, D.R., Sitarenios, G., 2001. Emotional intelligence as a standard intelligence. Emotion 1, 232-242.

Mayer, J.D., Salovey, P., Caruso, D.R., 2002. Mayer-Salovey-Caruso Emotional Intelligence Test (MSCEIT): USER'S Manual. Multi-Health Systems, Inc., Toronto, Ontario.

Mayer, J.D., Salovey, P., Caruso, D.R., Sitarenios, G., 2003. Measuring emotional intelligence with the MSCEIT V2.0. Emotion 3, 97-105.

Mayer, J.D., Roberts, R.D., Barsade, S.G., 2008. Human abilities: emotional intelligence. Annual Rev. Psychol. 59, 507-536.

McPheeters, H.L., 1984. Statewide mental health outcome evaluation: a perspective of two southern states. Community Ment. Health J. 20, 44-55.

Miller, T.J., McGlashan, T.H., Woods, S.W., Stein, K., Driesen, N., Corcoran, C.M., Hoffman, R., Davidson, L., 1999. Symptom assessment in schizophrenic prodromal states. Psychiatr. Q. 70 (4), 273-287.

Mischel, W., 1973. Toward a cognitive social learning reconceptualization of personality. Psychol. Rev. 80 (4), 252-283.

Mueser, K.T., Doonan, R., Penn, D.L., Blanchard, J.J., Bellack, A.S., Nishith, P., DeLeon, J., 1996. Emotion recognition and social competence in chronic schizophrenia. J. Abnorm. Psychology 105, 271-275.

Ochner, K.N., Phelps, E., 2007. Emerging perspectives on emotion-cognition interactions. Trends Cogn. Sci. 11 (8), 317-318.

Penn, D.L., Spaulding, W., Reed, D., Sullivan, M., 1996. The relationship of social cognition to ward behavior in chronic schizophrenia. Schizophr. Res. 20, 327-335.

Penn, D.L., Addington, J., Pinkham, A., 2006. Social cognitive impairments. In: Lieberman, J.A., Stroup, T.S., Perkins, D.O. (Eds.), American Psychiatric Association Textbook of Schizophrenia. American Psychiatric Publishing Press, Inc., Arlington, VA, pp. 261-274.

Poole, J.H., Tobias, F.C., Vinogradov, S., 2000. The functional relevance of affect recognition errors in schizophrenia. J. Int. Neuropsychol. Soc. 6, 649-658.

Reis, D.L., Brackett, M.A., Shamosh, N.A., Kiehl, K.A., Salovey, P., Gray, I., 2007. Emotional intelligence predicts individual differences in social exchange reasoning. Neuroimage 35, 1385-1391.

Rode, J.C., Mooney, C.H., Arthaud-Day, M.L., Near, J.P., Baldwin, T.T., Rubin, R.S., Bommer, W.H., 2007. Emotional intelligence and individual performance: evidence of direct and moderated effects. J. Org. Behav. 28, 399-421.

Salovey, P., Grewal, D., 2005. The science of emotional intelligence. Curr. Dir. Psychol. Sci. 14, 281-285.

Salovey, P., Mayer, J.D., 1990. Emotional intelligence. Imagin. Cogn. Pers. 9, 185-211.

Schneider, F., Gur, R.C., Gur, R.E., Shtasel, D.L., 1995. Emotional processing in schizophrenia: neurobehavioral probes in relation to psychopathology. Schizophr. Res. 17, 67-75.

Sergi, M.J., Rassovsky, Y., Nuechterlein, K.H., Green, M.F., 2006. Social perception as a mediator of the influence of early visual processing on functional status in schizophrenia. Am. J. Psychiatr. 163, 448-454.

Streit, M., Gaebel, W., Wolver, W., 1997. Facial-affect recognition and visual scanning behaviour in the course of schizophrenia. Schizophr. Res., 24, 311-317.

Sweet, L.H., Primeau, M., Fichtner, C.G., Lutz, G., 1998. Dissociation of affect recognition and mood state from blunting in patients with schizophrenia. Psychiatr. Res. 81, 301-308.

Vauth, R., Rusch, N., Wirtz, M., Corrigan, P.W., 2004. Does social cognition influence the relation between neurocognitive deficits and vocational functioning in schizophrenia? Psychiatr. Res. 128, 155-165. 